The Egyptian International Journal of Engineering Sciences \& Technology, Vol 9, No 1 (2005)

\title{
Learning System Approach
}

AbdelRazek AbouEINoor, louay AbouEINour

\author{
Abstract \\ Conventional Teaching vs Educational Technology (CT vs ET)t
}

